\title{
INFLUÊNCIA DO MÉTODO DE SÍNTESE PARA A PREPARAÇÃO DE NANOPARTÍCULAS DE TITANATO DE BÁRIO
}

\author{
L.R. PRADO ${ }^{1}$, N. S. de RESENDE ${ }^{2}$,R. S.SILVA ${ }^{3}$, S.M.S. EGUES ${ }^{1}$, G.R.SALAZAR-BANDA ${ }^{1}$ \\ ${ }^{1}$ Universidade Tiradentes, Instituto Tecnologia e Pesquisa \\ ${ }^{2}$ Universidade Federal do Rio de Janeiro/COPPE, Departamento de Química \\ ${ }^{3}$ Universidade Federal de Sergipe, Departamento de Física \\ E-mail para contato: gianrsb@gmail.com
}

\begin{abstract}
RESUMO - Neste trabalho foram desenvolvidas nanopartículas de titanato de bário por diversos métodos de síntese com o objetivo de comparar e verificar as características das nanopartículas sintetizadas pelos diversos métodos. A síntese foi realizada por quatro métodos distintos: método dos (Pechini), síntese eletroquímica, síntese hidrotérmica e hidrotérmica assistida por micro-ondas. Análises de difratometria de raios X evidenciaram a formação de titanato de bário na fase cúbica. As nanopartículas sintetizadas apresentaram área superficial BET variando entre 10 a $15 \mathrm{~m}^{2} \mathrm{~g}^{-1}$. O método Pechini propiciou a formação de partículas bem menores do que os outros métodos, entre 23 a $30 \mathrm{~nm}$. Já o método de síntese eletroquímico levou à obtenção de partículas entre 40 e 174 nm, com ampla distribuição de tamanho de partícula. O emprego dos métodos hidrotérmicos conduziram a diferentes distribuições de tamanho de partícula, na síntese hidrotérmica convencional formaram-se partículas entre 80 a $200 \mathrm{~nm}$ e a síntese assistida por micro-ondas levou à formação de partículas variando entre 33 e $55 \mathrm{~nm}$ em tempos de reação menores do que aqueles usados na síntese usando aquecimento convencional. A energia de band gap de todas as nanopartículas sintetizadas determinada por refletância difusa no UV-vis foi em torno de $3,50 \mathrm{eV}$.
\end{abstract}

\section{INTRODUÇÃO}

O titanato de bário $\left(\mathrm{BaTiO}_{3}\right)$ foi descoberto na década de 40 quando foi relatada a característica de sua alta constante dielétrica em várias partes do Akdogan et al. (1999). O $\mathrm{BaTiO}_{3}$ pertence à família das perovskitas, do tipo $\mathrm{ABO}_{3}($ Kishi et al.,2003) e a sua estrutura tem uma baixa temperatura de Curie de aproximadamente $120{ }^{\circ} \mathrm{C}$, o que faz com que o material apresente constante dielétrica elevada à temperatura ambiente $\left(\varepsilon=3600\right.$ a $25^{\circ} \mathrm{C}$ e $10^{5}$ $\mathrm{Hz}$ ). Esta característica permite a sua utilização em capacitores de alta capacidade de armazenamento de carga (Lee et al., 2003;Chung et al., 2009). $\mathrm{O} \mathrm{BaTiO}_{3}$ apresenta estruturas de fases diferentes e que frequentemente exibem propriedades dielétricas distintas. Podem ocorrer três transições de fase no $\mathrm{BaTiO}_{3}$, em função da temperatura: de romboédrica para ortorrômbica, transição que ocorre em torno de $-90{ }^{\circ} \mathrm{C}$; de ortorrômbica para tetragonal, mudança de fase que ocorre a cerca de $5{ }^{\circ} \mathrm{C}$; e de tetragonal para cúbica em temperaturas de aproximadamente $120{ }^{\circ} \mathrm{C}$ (Cohen, 1992). Devido às suas excelentes propriedades piezoelétricas (ocorre polarização com aplicação de pressão) o $\mathrm{BaTiO}_{3}$ é utilizado no desenvolvimento de contadores, registradores, detectores de som, transdutores, bem como na fabricação de dispositivos microeletrônicos (Yashima et al.,2005). 
A síntese hidrotérmica para a preparação de $\mathrm{BaTiO}_{3}$ cristalino envolve reações químicas entre uma solução concentrada de hidróxido de bário e o $\mathrm{TiO}_{2}$, ou géis de misturas de acetato de $\mathrm{Ba}-\mathrm{Ti}$, a temperaturas relativamente baixas $\left(<1200{ }^{\circ} \mathrm{C}\right)$. Este método ganhou notoriedade devido à possibilidade de produzir partículas pequenas $(1$ a $1000 \mathrm{~nm})$ com distribuição de tamanho mais uniforme(Sasirekha et al.,2008). A interação entre as fases sólida e fluida permite controlar o tamanho das partículas através da adequação dos parâmetros de síntese, tais como os valores de $\mathrm{pH}$, a temperatura da reação e o tempo de aquecimento. $\mathrm{O}$ controle destes parâmetros pode estabilizar a formação das partículas de $\mathrm{BaTiO}_{3}$ e retardar a formação de impurezas. Além disso, os precursores para a preparação do $\mathrm{BaTiO}_{3}$ pela rota hidrotérmica são de baixo custo e fácil manuseio, o que torna este método simples e eficaz para a preparação de $\mathrm{BaTiO}_{3}($ Sasirekha et al.,2008).

A tecnologia de aquecimento por micro-ondas tem sido aplicada para reações orgânicas desde o final dos anos 80 (Sanseverino, 2002). Entretanto, não é um método comumente utilizado nos laboratórios de síntese de materiais. O mecanismo de ação das micro-ondas é o aquecimento dielétrico, seja por rotação de dipolo ou por condução iônica.A aplicação do aquecimento micro-ondas na síntese hidrotérmica, ocorre pela interação entre as micro-ondas e as substâncias polares do meio reacional: a radiação é absorvida pelo reagente e a energia eletromagnética é convertida em energia térmica. Dessa forma, o calor é gerado a partir do interior do material, o que permite uma redução do tempo de processamento e do custo energético (Agrawal,1998).

Por outro lado, a rota eletroquímica é de interesse considerável por causa de um controle do tamanho de partícula conseguido ajustando a densidade de corrente ou o potencial aplicado, que é considerado como o objeto mais atrativo para a síntese química neste campo. Na síntese eletroquímica, investigações extensivas foram centradas sobre partículas metálicas, especialmente em partículas de metais nobres, quando somente alguns dos estudos foram executados na síntese de compostos inorgânicos (Jiang et al.,2004).

O método Pechini também conhecido como método dos precursores poliméricos, foi proposto por Pechini na década de 60, este método envolve a capacidade que alguns ácidos orgânicos hidrocarboxílicos possuem para formação de quelatos no qual estão incorporados os cátions metálicos distribuídos homogeneamente. A solução do sal no ácido é misturada com um álcool poli-hidroxilado, sob aquecimento e agitação, ocorre à formação de um éster por conta da condensação entre o álcool e o quelato ácido. Neste processo o álcool esterifica as moléculas do ácido carboxílico gerando água a qual é removida por evaporação. Já que tanto o ácido como o álcool são polifuncionais, ocorre a formação de uma resina polimérica (poliéster) com os cátions quelados distribuídos atomicamente ao longo da estrutura molecular da resina(Pechini, 1967).

Neste contexto, o objetivo deste trabalho foi sintetizar nanopartículas de titanato de bário por quatro métodos diferentes: (Pechini), eletroquímico, hidrotérmico e hidrotérmico assistido por micro-ondas. Estes métodos permitiram obter materiais com diferentes tamanhos de partícula, características estruturais e eletrônicas.

\section{SEÇÃO EXPERIMENTAL}

\subsection{Síntese de $\mathrm{BaTiO}_{3}$ pelo método hidrotérmico}


Os precursores hidróxido de bário octahidratado $\left(\mathrm{Ba}(\mathrm{OH})_{2} .8 \mathrm{H}_{2} \mathrm{O}\right)$ da Synthe partículas $\mathrm{TiO}_{2}$ P-25 Degussa. O hidróxido de bário foi dissolvido em água destilada e em seguida adicionado o dióxido de titânio com agitação durante 2 horas, foi obtida uma suspensão, e a mesma foi levada a um vaso de teflon dentro de uma autoclave de aço rosqueada, este é aquecido a uma temperatura de até $150^{\circ} \mathrm{C}$ e sob pressão autogênica, a estufa equipada com eixo giratório mantida sob agitação $(56 \mathrm{rpm})$ por $24 \mathrm{~h}$. A suspensão foi lavada com solução de $\mathrm{HNO}_{3}$ da Vetec $\left(0,1 \mathrm{~mol} \mathrm{~L}^{-1}\right)$ e água destilada, filtrada e seca a $120{ }^{\circ} \mathrm{C}$.

\subsection{Síntese de $\mathrm{BaTiO}_{3}$ pelo método hidrotérmico via micro-ondas}

A suspensão foi obtida seguindo a mesma metodologia usada no método hidrotérmico. Porém, neste caso, a mesma foi levada a um reator micro-ondas monomodo da CEM modelo Discover, as reações foram realizadas em batelada. A mistura foi mantida a temperatura constante $\left(170^{\circ} \mathrm{C}\right)$ sob agitação constante durante 4 horas em uma frequência de $2,45 \mathrm{GHz}$. Mesmo procedimento de lavagem e secagem do método anterior.

\subsection{Síntese de $\mathrm{BaTiO}_{3}$ pelo método eletroquímico}

A síntese de titanato de bário pelo método eletroquímico foi conduzida em uma célula eletroquímica com capacidade de $200 \mathrm{~mL}$ acoplada a um banho termostático (Nova Ética Banho Ultratermostático), usada com a finalidade de manter a temperatura da reação em aproximadamente $50{ }^{\circ} \mathrm{C}$. A reação na célula foi mantida sob agitação magnética e possuía eletrodos ligados a uma fonte Dupla 0-30 V 3A (ICEL Manaus PS - 5000), a qual pode trabalhar em série, obtendo-se assim um potencial máximo de $60 \mathrm{~V}$ e uma corrente de mais ou menos 3,25 A sendo assim, suficiente desprender o titânio do eletrodo e então formar o $\mathrm{BaTiO}_{3}$ numa reação de 2 horas.

Como eletrólito foram utilizados $200 \mathrm{~mL}$ de soluções com concentração de $0,1 \mathrm{~mol} \mathrm{~L}^{-1}$ de hidróxido de bário octahidratado $\left(\mathrm{Ba}(\mathrm{OH})_{2} .8 \mathrm{H}_{2} \mathrm{O}\right)$ da Synth e $1 \mathrm{~mol} \mathrm{~L}^{-1}$ de hidróxido de sódio $99 \%(\mathrm{NaOH})$ da Vetec. Dissolveu-se primeiramente o $\mathrm{Ba}(\mathrm{OH})_{2}$, em seguida foi adicionado o $\mathrm{NaOH}$ sob agitação constante. Seguidamente fez-se o tratamento dos eletrodos de titânio, assim, estes foram lixados e lavados com acetona e água destilada. Cada eletrodo medindo aproximadamente $7 \mathrm{~cm} \times 2 \mathrm{~cm}$ de comprimento e largura.

As partículas foram lavadas abundantemente com água destilada e em seguida filtradas a vácuo, inicialmente foi usada uma solução aquosa $0,1 \mathrm{~mol} \mathrm{~L}^{-1}$ de ácido nítrico para analisar a eficiência na remoção do carbonato de cada uma destas soluções. Finalmente as partículas foram lavadas novamente e secas na estufa a uma temperatura de $120^{\circ} \mathrm{C}$.

\subsection{Síntese de $\mathrm{BaTiO}_{3}$ pelo método Pechini}

Foram utilizados o ácido cítrico $\left(\mathrm{C}_{6} \mathrm{H}_{8} \mathrm{O}_{7}\right)$ da Synth acetato de bário $\left(\mathrm{BaC}_{4} \mathrm{H}_{6} \mathrm{O}_{4}\right)$ da Synth e o etileno glicol $\left(\mathrm{C}_{2} \mathrm{H}_{6} \mathrm{O}_{2}\right)$ da Vetec, para a formação das soluções precursoras.

- Procedimento de preparo do citrato de bário com etileno glicol:

Inicialmente foram dissolvidas 10,9831 g de acetato de bário em aproximadamente 400 $\mathrm{mL}$ de água destilada. Paralelamente, foram dissolvidos $24,7848 \mathrm{~g}$ de ácido cítrico, para seguidamente misturar as duas soluções e adicionar $16,5232 \mathrm{~g}$ de etileno glicol; sendo esta mistura deixada sob agitação até completa dissolução. 
- Procedimento para preparo do citrato de titânio com etileno glicol:

As soluções de citrato de titânio foram preparadas dissolvendo-se 80,6946 g de ácido cítrico em água destilada. Após esse procedimento, foram adicionados lentamente $20 \mathrm{~g}$ de isopropóxido de titânio, logo em seguida foram acrescentados 53,7964 g de etileno glicol e dissolvidos sob agitação constante.

Logo, os dois complexos preparados misturaram-se e se ajustou o $\mathrm{pH}$ em 9 usando $\mathrm{NH}_{4} \mathrm{OH}$ e em seguida deixou-se sob agitação magnética e aquecendo a uma temperatura de $200{ }^{\circ} \mathrm{C}$, até formar uma solução de cor amarelada e aspecto viscoso. Logo depois esta solução foi calcinada em um forno durante $5 \mathrm{~h}$ a $600{ }^{\circ} \mathrm{C}$ sob a taxa de aquecimento de $2{ }^{\circ} \mathrm{C} \mathrm{min}^{-1}$. Assim, foi obtido um pó de cor branca, bastante fino, que foi em seguida macerado e armazenado.

\section{RESULTADOS E DISCUSSÃO}

\subsection{Análises por difração de raios $X$ (DRX)}

Os difratogramas de raios $\mathrm{X}$ das nanopartículas sintetizadas pelos métodos de síntese hidrotérmico, hidrotérmico micro-ondas, Pechini e eletroquímico podem ser observados na Figura 1. Os picos identificados no difratograma das amostras sintetizadas via rota eletroquímica são característicos do $\mathrm{BaTiO}_{3}$ na estrutura cúbica de acordo com o banco de dados do JCPDS 31-0174. Pode-se observar também a formação de pequenas quantidades de carbonato de bário (JCPDS 05-0378) na fase witherite que pode ser produzido a partir do $\mathrm{CO}_{2}$ atmosférico.

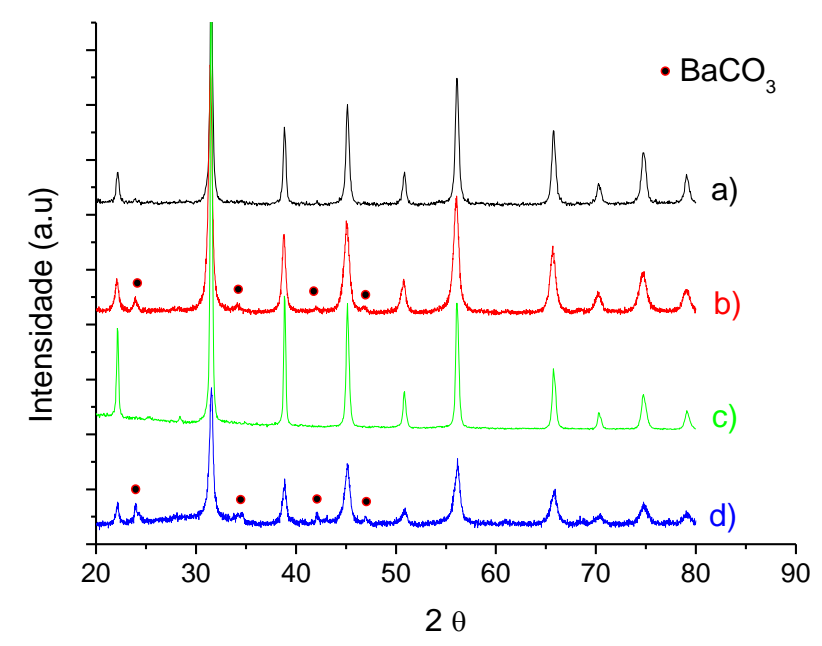

Figura 1-Difratogramas de raios $X$ das amostras sintetizadas pelas rotas: $a)$ micro-ondas, $b$ ) Pechini, c) hidrotérmico e d) eletroquímica.

No difratograma da Figura 1 observa-se que as amostras sintetizadas pelo método Pechini também apresentaram a formação de carbonato de bário, porém não foi realizado nenhum tipo de lavagem para a retirada do mesmo nesta amostra. Neste caso também foi 
identificado o titanato de bário de estrutura perovskita cúbica simples de acordo com o JCPDS 31-0174. Neste sentido, Chen E Chen,(2004) sintetizaram titanato de bário pelo método hidrotérmico e observaram que a formação de $\mathrm{BaCO}_{3}$ (JCPDS:41-0373) sem nenhum tipo de lavagem, em seguida eles lavaram com ácido fórmico e removeram toda fase indesejada.

Já no difratograma de raios $\mathrm{X}$ referente à amostra sintetizada pelo método hidrotérmico, no qual foi realizada uma lavagem do pó com solução de ácido nítrico $\left(0,1 \mathrm{~mol} \mathrm{~L}^{-1}\right)$. Após a lavagem foi obtido apenas o pó de titanato de bário puro e foi identificada a estrutura perovskita cúbica simples de acordo com o JCPDS 31-0174, mostrando que o ácido nítrico é eficiente na remoção de $\mathrm{BaCO}_{3}$.

\subsection{Medidas de áreas de superficie específicas das nanopartículas (BET)}

$\mathrm{Na}$ Tabela 1 estão listados os resultados das medidas de área superficial das partículas sintetizadas pelos diferentes métodos.

Tabela 1 -Área superficial específica das nanopartículasde $\mathrm{BaTiO}_{3}$.

\begin{tabular}{|c|c|c|c|c|}
\hline Método de Síntese & Hidrotérmico & Micro-ondas & Eletroquímico & Pechini \\
\hline $\mathbf{S}_{\text {BET }}\left(\mathbf{m}^{\mathbf{2}} \mathbf{g}^{-\mathbf{1}}\right)$ & $<10.0$ & 12.0 & 13.5 & 15.0 \\
\hline
\end{tabular}

Podemos observar na Tabela 1 que a área superficial dos pós-sintetizados pelos quatro métodos. No trabalho de Mandal(2007), foi realizada a síntese de titanato de bário através do método (Pechini) com os mesmo precursores e em condições de preparo semelhantes às deste trabalho com temperaturas de $400-600{ }^{\circ} \mathrm{C}$ por 2 horas e obtiveram como resultados da média da área superficial em torno de $15-20 \mathrm{~m}^{2} \mathrm{~g}^{-1}$, resultados que se assemelha com os encontrados neste trabalho.

Podemos observar no trabalho de Chen e Chen (2003) que área superficial em torno de 7,6-11,2 $\mathrm{m}^{2} \mathrm{~g}^{-1}$ é encontrada para partículas de titanato de bário preparadas usando o $\mathrm{TiO}_{2}$ (Degussa) como precursor e condições de síntese hidrotérmica de $85-180{ }^{\circ} \mathrm{C}$ por 24 horas. As nanopartículas sintetizadas pelo método hidrotérmico via micro-ondas, com tempo de reação de 4 horas a uma temperatura de $170{ }^{\circ} \mathrm{C}$ apresentaram área superficial de $12 \mathrm{~m}^{2} \mathrm{~g}^{-1}$. Resultado semelhante ao encontrado por Nyutu et al.(2008) que sintetizaram nanopartículas de titanato de bário usando $\mathrm{BaCl}_{2} \cdot 2 \mathrm{H}_{2} \mathrm{O}$ e isopropóxido de titânio, utilizando frequência de $2,45 \mathrm{GHz}$ e temperatura de $170{ }^{\circ} \mathrm{C}$ por 10 horas, obtendo partículas com $12 \mathrm{~m}^{2} \mathrm{~g}^{-1}$, foi comprovado neste estudo que não são necessárias 10 horas para obter o titanato de bário com a mesma área superficial.

\subsection{Microscopia Eletrônica de Varredura (MEV)}

A Figura 2- a) mostra imagens da amostra sintetizada pelo método eletroquímico onde é possível identificar partículas quase esféricas e alguns bastonetes. Segundo Lopez et al. (1999) estes bastonetes são constituídos de carbonato de bário. Nas micrografias também se 
observa que as nanopartículas apresentam-se bastante aglomeradas e com diâmetro de partícula na faixa entre aproximadamente 40 e $175 \mathrm{~nm}$.

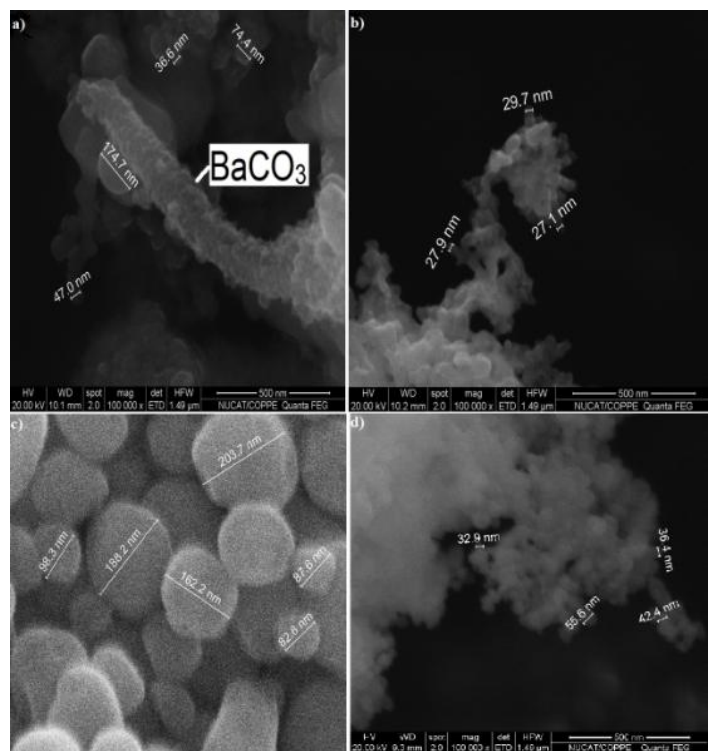

Figura 2 - a) Imagens de MEV-FEG das nanopartículas sintetizadas pelo método eletroquímico, b) pelo método Pechini, c) pelo método hidrotérmico e d) método hidrotérmico com aquecimento de micro-ondas com aproximação de 100.000×.

Na Figura 2 - b) pode-se observar as amostras sintetizadas pelo método dos (Pechini) e nelas podemos identificar grãos homogêneos e parcialmente aglomerados. De acordo com Silva et al. (2007) o pH tem uma influência significativa na morfologia dos pós sintetizados e quando o $\mathrm{pH}>7$ a tendência é que as partículas sejam mais homogêneas e menos aglomeradas. As partículas sintetizadas pelo método Pechini são as que apresentaram menores dimensões, quando comparadas aos outros métodos de síntese deste trabalho, em torno de 23 a $30 \mathrm{~nm}$. Resultados estes semelhantes aos de Vinothini et al.(2006), que sintetizaram titanato de bário usando a rota Pechini e obtiveram partículas de $20 \mathrm{~nm}$ na fase cúbica com alguns traços da fase hexagonal.

Já na Figura 2 - c) podemos observar partículas bem dispersas, homogêneas e esféricas obtidas a partir da síntese pelo método hidrotérmico, porém com tamanho médio de partículas variando entre 80 e $200 \mathrm{~nm}$. Segundo Sasirekha et al.(2008), que utilizaram temperaturas de síntese entre 80 até $200{ }^{\circ} \mathrm{C}$, o aumento na temperatura de síntese leva a um aumento do tamanho das partículas.

Na Figura 2 - d) encontram-se partículas esféricas em escala nanométrica e aglomeradas com tamanho de partícula variando entre 33 e $55 \mathrm{~nm}$. Komarneni e Katsuki,(2010) estudaram a síntese de titanato de bário com e sem agitação e sob radiação micro-ondas, variaram a temperatura reacional entre 150 e $200{ }^{\circ} \mathrm{C}$, usando um tempo de $4 \mathrm{~h}$. Quando utilizaram agitação e temperatura de $180{ }^{\circ} \mathrm{C}$ obtiveram partículas de aproximadamente $30 \mathrm{~nm}$ já em condições estáticas formaram partículas aglomeradas e maiores de entre 60-100 nm.

\subsection{Espectroscopia com Refletância Difusa no UV-Visível}


Na Tabela 2 estão apresentados os valores estimados da energia de band gap das nanopartículas sintetizadas pelos métodos eletroquimico, Pechini, hidrotérmico e hidrotérmico via micro-ondas.

Tabela 2 - Valores estimados para a energia de band gap para os materiais sintetizados pelos diferentes métodos.

\begin{tabular}{|c|c|c|c|c|}
\hline Método de Síntese & Hidrotérmico & Micro-ondas & Eletroquímico & Pechini \\
\hline $\begin{array}{c}\text { Gap de energia } \\
\text { (eV) }\end{array}$ & 3,55 & 3,55 & 3,56 & 3,54 \\
\hline Absorção (nm) & 349 & 349 & 348 & 350 \\
\hline
\end{tabular}

No trabalho de Zieliskaa e Morawski, (2005) estes autores obtiveram nanopartículas de $\mathrm{BaTiO}_{3}$ com energia de band gap de $3,33 \mathrm{eV}$ que está relativamente próxima às energias calculadas neste trabalho; isso implica que nestes materiais as propriedades fotocatalíticas podem existir sob a irradiação da luz ultravioleta.

\section{CONCLUSÕES}

Neste trabalho estudou-se a síntese de nanopartículas de titanato de bário pelos métodos: dos (Pechini), eletroquímico, hidrotérmico e hidrotérmico com aquecimento microondas. Comparando os quatro métodos a síntese via micro-ondas se destacou por constituir nanopartículas de titanato de bário em menor tempo de reação e o que mais apresentou facilidades operacionais,porém o método Pechini por formar partículas menores, o eletroquímico por utilizar temperaturas de reação menores e o hidrotérmico por formar partículas menos aglomeradas.

\section{REFERENCIAS}

AGRAWAL, D.K.; Microwave Processing of Ceramic. Curr. Opin. Solid State Mater. Sci. v. 3, p 480-485,1998.

AKDOGAN, E. K; LEONARD, M. R; SAFARI, A. in Handbook of Low and High Dielectric Constant Materials and Their Applications.San Diego. Ed. Hari Singh Nalwa, 1999.

CHEN, H-J; CHEN, Y-W. Hydrothermal Synthesis of Barium Titanate. Ind. Eng. Chem. Res. v. 42, p. 473-483, 2003.

CHEN, K-Y.; CHEN, Y-W. Preparation of barium titanate ultrafine particles from rutile titania by a hydrothermal conversion. Powder Technol. v. 141, p 69-74, 2004.

CHUNG, U. C; ELISSALDE, C; MORNET, S; MAGLIONE, M; ESTOURNES C. Controlling internal barrier in low loss $\mathrm{BaTiO}_{3}$ supercapacitors. Appl. Phys. Lett. v.94, p 072903,2009.

COHEN,R. E. Origin of ferroelectricity in perovskite oxides.Nature (London) v.358, p. $136-138,1992$. 
JIANG, K., HU, X. H., SUN, H. J., WANG,D . H., JIN, X . B., Y. Y. REN AND G.Z. CHE. Electrochemical Synthesis of $\mathrm{LiTiO}_{2}$ and. LiTiO in Molten LiCl. Chem. Mater., v. 16, p. 4324-4329, 2004.

KISHI, H; MIZUNO, Y; CHAZONO, H. Base-Metal Electrode-Multilayer Ceramic Capacitors: Past, Present and Future Perspectives. Jpn. J. Appl. Phys. v.42, p 1-15, 2003.

KOMARNENI, S., KATSUKI, H. Microwave-hydrothermal synthesis of barium titanate under stirring condition.Ceram. Int., v.36, p. 1165-1169, 2010.

LEE, J-Y;LEE, J-H; HONG, S-H;LEE, Y.K; CHOI, J-Y: Coating of $\mathrm{BaTiO}_{3}$ nanolayer on spherical Ni powder for MLCC. Adv. Mater. v.15, p. 1655-1657, 2003.

LOPEZ, M. C. B.; FOURLARIS, G.; RAND, B.; RILEY, F. L.; Characterization of Barium Titanate Powders: Barium Carbonate Identification. J. Am. Ceram. Soc. v. 82, p. 1777-1786, 1999.

MANDAL, T.K. Characterization of tetragonal $\mathrm{BaTiO}_{3}$ nanopowders prepared with a new soft chemistry route. Mater. Lett. v. 61, p. 850-854, 2007.

NYUTU, E. K., CHEN, CHUN-CHUN-HU., DUTTA, P. K., SUIB, S. L. Effect of Microwave Frequency on Hydrothermal Synthesis of Nanocrystalline Tetragonal Barium Titanate. J. Phys. Chem. C. v.112, p.9659-9667, 2008.

PECHINI, M. Method of Preparing Lead and Alkaline Earth Titanates and Niobates and Coating Method using the same from a Capacitor. U. S. Patent. $\mathrm{N}^{\circ} 3330697,1967$.

SANSEVERINO, A.M. Micro-ondas em síntese Orgânica . Quim. Nova.v. 25,p. 660667, 2002.

SASIREKHA, N; RAJESH, B; CHEN, Y-W. Hydrothermal Synthesis of Barium Titanate: Effect of Titania Precursor and Calcination Temperature on Phase Transition.Ind. Eng. Chem. Res. v. 47, p. 1868-1875, 2008.

SILVA, R. S., BERNARDI, M.I.B., HERNANDES, A. C. Synthesis of nonagglomerated $\mathrm{Ba}_{0.77} \mathrm{Ca}_{0.23} \mathrm{TiO}_{3}$ nanopowders. J. Sol-Gel Sci. Technol. v. 42, p. 173-179, 2007.

VINOTHINI, V.; SINGH, P.; BALASUBRAMANIAN, M. Synthesis of barium titanatenanopowder using polymeric precursor method. Ceram. Int. v. 32, p. 99-103, 2006.

YASHIMA, M; HOSHINA, T.; ISHIMURA, D.; KOBAYASHI, S.; NAKAMURA,W.; TSURUMI, T.; WADA, S.Size effect on the crystal structure of barium titanate nanoparticles.J. Appl. Phys. v.98, p. 014313, 2005.

ZIELISKAA, B e MORAWSKI, A.W. TiO 2 photocatalysts promoted by alkali metals.Appl. Catal. B. v. 55, p. 221-226, 2005. 